\title{
Alterations in AQP4 expression and polarization in the course of motor neuron degeneration in SOD1G93A mice
}

\author{
JIAYING DAI ${ }^{1}$, WEIHAO LIN ${ }^{2 *}$, MINYING ZHENG $^{1}$, QIANG LIU $^{3}$, BAIXUAN HE $^{1}$,

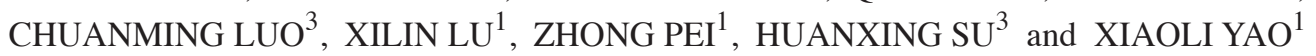 \\ ${ }^{1}$ Department of Neurology, National Key Clinical Department and Key Discipline of Neurology; \\ ${ }^{2}$ Department of Breast and Thyroid Surgery, The First Affiliated Hospital, Sun Yat-sen University, \\ Guangzhou, Guangdong 510080; ${ }^{3}$ State Key Laboratory of Quality Research in Chinese Medicine, \\ Institute of Chinese Medical Sciences, University of Macau, Macau SAR 999078, P.R. China
}

Received May 31, 2016; Accepted April 10, 2017

DOI: $10.3892 / \mathrm{mmr} .2017 .6786$

\begin{abstract}
Amyotrophic lateral sclerosis (ALS) is a progressive neurodegenerative disease characterized by selective degeneration of upper and lower motor neurons. The disease progression is associated with the astrocytic environment. Aquaporin-4 (AQP4) water channels are the most abundant AQPs expressed in astrocytes, exerting important influences on central nervous system homeostasis. The present study aimed to characterize the alterations in AQP4 expression and localization in superoxide dismutase 1 (SOD1) G93A transgenic mice. SOD1G93A mice were sacrificed during the presymptomatic, disease onset and end stages and immunostaining was performed on spinal cord sections to investigate neuronal loss, glial activation and AQP4 expression in the spinal cord. It was observed that global AQP4 expression increased in the spinal cord of SOD1G93A mice as the disease progressed. However, AQP4 polarization decreased as the disease progressed, and AQP4 polarized localization at the endfeet of astrocytes was decreased in the spinal ventral horn of SOD1G93A mice at the disease onset and end stages. Meanwhile, motor neuron degeneration and decreased glutamate transporter 1 expression in
\end{abstract}

Correspondence to: Professor Xiaoli Yao, Department of Neurology, National Key Clinical Department and Key Discipline of Neurology, The First Affiliated Hospital, Sun Yat-sen University, 58 Sec Zhongshan Road, Guangzhou, Guangdong 510080, P.R. China E-mail: 1iliyao71@163.com

Dr Huanxing Su, State Key Laboratory of Quality Research in Chinese Medicine, Institute of Chinese Medical Sciences, University of Macau, N22 Building, University Great Road, Macau SAR 999078, P.R. China

E-mail: huanxingsu@umac.mo

*Contributed equally

Key words: amyotrophic lateral sclerosis, astrocyte, aquaporin-4 polarization, glutamate transporter 1 , superoxide dismutase $1 \mathrm{G} 93 \mathrm{~A}$ mice astrocytes in SOD1G93A mice were observed as the disease progressed. The results of the present study demonstrated that AQP4 depolarization is a widespread pathological condition and may contribute to motor neuron degeneration in ALS.

\section{Introduction}

Amyotrophic lateral sclerosis (ALS) is a progressive and fatal neurodegenerative disease, characterized by selective degeneration and death of motor neurons in the motor cortex, brainstem and spinal cord. A total of $~ 90 \%$ of ALS cases are sporadic (sALS), while $10 \%$ are familial (fALS) (1). The clinical presentation and pathology of sALS and fALS are similar. The superoxide dismutase 1 (SOD1) gene, encoding $\mathrm{Cu} / \mathrm{Zn}$ superoxide dismutase, was the first identified ALS risk gene. Mutations in SOD1 account for $20 \%$ of fALS and $3 \%$ of sALS cases (2). However, the precise mechanisms causing motor neuron degeneration in ALS remain unknown. Several mechanisms have been investigated, including oxidative stress, mitochondrial dysfunction, excitotoxicity, endoplasmic reticulum stress and neuroinflammation (3). In addition, the involvement of blood-brain barrier (BBB) and blood-spinal cord barrier (BSCB) dysfunction in ALS has been a focus of research.

The BBB and BSCB have important roles in controlling the homeostasis of water and other substances in the central nervous system (CNS), by transporting substances selectively through the systemic compartment and preventing passive diffusion of harmful blood solutes. A number of studies have discovered that the BSCB is impaired patients with fALS and sALS (4-7). In addition, similar BSCB impairment was observed in SOD1 mutant ALS animal models. Previous studies demonstrated capillary ultrastructure alterations and BSCB damage in SOD1G93A transgenic mice (mice carrying a mutant human SOD1 cDNA inserted randomly into the mouse genome) at the early and late stages of the disease $(8,9)$; the direct effects of the SOD1 mutation on APQ protein function are currently unknown. Recently, a study demonstrated that restoration of BSCB integrity at an early disease stage delayed the onset of motor neuron impairment and degeneration in ALS (10). 
Astrocytes are important components of the CNS. It has been demonstrated that astrocytes are associated with disease onset and progression in ALS (11). Astrocytic endfeet wrap around the blood vessel wall and form parts of the BSCB. In addition, astrocytes serve primary roles in maintaining the homeostasis of water, glutamate and ions in the CNS, depending on specific molecules expressed in astrocytes, including water channel protein aquaporin-4 (AQP4), glutamate transporter 1 (GLT-1 in rodents, EAAT2 in humans) and potassium inwardly-rectifying channel, Kir4.1.

AQP4 water channels are the most abundant AQPs expressed in the CNS, permitting passive and bidirectional water diffusion. Several studies have demonstrated that AQP4 is involved in BBB integrity and perturbation (12). In ovariectomized animals, AQP4 expression was demonstrated to be increased following disruption of the BBB by intraparenchymal injection of lipopolysaccharide (13). A recent study demonstrated that remote ischemic post-conditioning was able to alleviate brain edema and BBB permeability by downregulating AQP4 (14). In addition, accumulating evidence indicates that AQP4 serves an important role in neurological disorders, including cerebral ischemia (14), epilepsy (15), traumatic brain injury (16), Alzheimer's disease (17) and spinal cord injury (18).

AQP4 overexpression has been observed in the brain and spinal cord of SOD1G93A ALS models rodent (19-21), suggesting that AQP4 may contribute to motor neuron degeneration in ALS. The function of AQP4 is dependent upon perivascular AQP4 polarization, which refers to that AQP4 that is localized primarily in perivascular astrocytic endfeet domains. The function of AQP4 overexpression in ALS has not been elucidated. Specifically, it remains to be investigated whether ALS is associated with a loss of AQP4 polarization. In the present study, alterations in AQP4 distribution were investigated in ALS animal models.

\section{Materials and methods}

Animals. Transgenic mice carrying human G93A mSOD1 [strain B6SJL-Tg (SOD1-G93A) 1Gur/J] were obtained from the Jackson Laboratory (Ben Harbor, ME, USA) and crossed with female mice with a B6SJL/F1 background for $\geq 4$ generations. Transgenic offspring were genotyped using polymerase chain reaction analysis of DNA obtained from tail biopsies, as described previously (22). According to the scoring system used to evaluate signs of motor deficit in SOD1G93A mice, animals were divided into three stages: i) Presymptomatic stage (60 days) with no sign of motor dysfunction; ii) disease onset stage with hind limb tremors observed when suspended by the tail, followed by gait abnormalities; iii) end-stage, animal unable to right itself within $30 \mathrm{sec}$ (23).

A total of 20 adult male mice (weight, $18-25 \mathrm{~g}$; age, 8-10 weeks) were housed in a $12 / 12 \mathrm{~h}$ light-dark cycle and a controlled temperature $\left(23 \pm 2^{\circ} \mathrm{C}\right)$, with free access to food and water. All procedures performed on the animals were approved by the Animal Ethical Committee at Sun Yat-sen University (Guangzhou, China), and were in accordance with the Guidelines for the Care and Use of Laboratory Animals of the National Institutes of Health (Bethesda, MA, USA; publication no. 80-23; revised in 1996).
Immunofluorescence. Transgenic and wild type (WT) mice were deeply anesthetized with $1 \%$ pentobarbital $(50 \mathrm{mg} / \mathrm{kg}$ intraperitoneally) and transcardially perfused with $0.9 \%$ normal saline, followed by $4 \%$ paraformaldehyde in $0.1 \mathrm{~mol} / 1 \mathrm{PBS}$ ( $\mathrm{pH}$ 7.4). The spinal cords were dissected to identify the cervical and lumbar segments, further post-fixed in $4 \%$ paraformaldehyde at $4{ }^{\circ} \mathrm{C}$ overnight, and immersed in 20 and $30 \%$ sucrose for 2 days at $4^{\circ} \mathrm{C}$. Tissues were cut into $20 \mu \mathrm{m}$ frozen sections. Antigen retrieval was conducted on the selected sections, which were heated in the microwave for $7 \mathrm{mins}$ in $0.01 \mathrm{~mol} / 1$ citric acid solution ( $\mathrm{pH}$ 6.0). Following washing with $0.01 \mathrm{M}$ PBS for 5 mins, the sections were blocked with Immunol Staining Blocking Buffer (cat. no. P0102; Beyotime Institute of Biotechnology, Shanghai, China) for $1 \mathrm{~h}$ at room temperature. The sections were then washed three times with 0.01 M PBS for $5 \mathrm{~min}$ in an orbital shaker prior to incubation with primary antibodies. Primary antibodies included goat anti-mouse choline O-acetyltransferase (ChAT) polyclonal antibody (cat. no. AB144P; EMD Millipore, Billerica, MA, USA) at 1:400 dilution; mouse anti-mouse glial fibrillary acidic protein (GFAP) monoclonal antibody (cat. no. 3670; Cell Signaling Technology, Inc., Danvers, MA, USA) at 1:500 dilution; rabbit anti-mouse AQP4 polyclonal antibody (cat. no. AQP-014; Alomone Labs, Jerusalem, Israel) at 1:400 dilution; and rabbit anti-mouse GLT-1 polyclonal antibody (cat. no. ab106289; Abcam, Cambridge, UK) at 1:300 dilution, overnight at $4^{\circ} \mathrm{C}$. The sections were washed three times with $0.01 \mathrm{~mol} / 1 \mathrm{PBS}$ for $5 \mathrm{~min}$ in an orbital shaker, and incubated for $1 \mathrm{~h}$ at room temperature with the appropriate species-specific fluorophore-conjugated secondary antibodies: Donkey anti-goat IgG Alexa Fluor ${ }^{\circledR} 594$ (cat. not. A-11058; Invitrogen; Thermo Fisher Scientific, Inc., Waltham, MA, USA; 1:400) for ChAT; goat anti-mouse IgG Alexa Fluor ${ }^{\circledR} 555$ (cat. no. 4409; Cell Signaling Technology, Inc.; 1:400) for GFAP; anti-rabbit IgG Alexa Fluor ${ }^{\circledR} 488$ (cat. no. 4412; Cell Signaling Technology, Inc.; 1:400) for AQP4 and GLT-1. Following washing with $0.01 \mathrm{~mol} / 1 \mathrm{PBS}$, the sections were mounted using Fluoroshield Mounting Medium with DAPI (Sigma-Aldrich; Merck KGaA, Darmstadt, Germany).

Analysis of motor neuron survival in the spinal cord. In order to analyze the survival of motor neurons in the cervical and lumbar spinal cord, the total number of ChAT-expressing cells was counted in every fourth section (in 5 animals/group). ChAT-expressing cells were counted if they exhibited a nucleolus that was located in the ventral horns of immunoreacted sections. The number of motor neurons in the ventral horn of the WT animal was used as the control value. The number of surviving motor neurons in the ventral horn of SOD1G93A mice was described quantitatively as percentages of the control value. Imaging was conducted at $\mathrm{x} 20$ objective power using a fluorescence microscope (Olympus DP70; Olympus Corporation, Tokyo, Japan).

Evaluation of GFAP and AQP4 expression and localization. Free-floating immunofluorescence staining of $20 \mu \mathrm{m}$ fixed cross-spinal sections was performed to evaluate the protein expression patterns of AQP4 and GFAP. Imaging was conducted at a low magnification (x20 objective power) and at a high magnification (x63 oil-immersion objective power using a laser scanning confocal microscope; SP5; Leica 


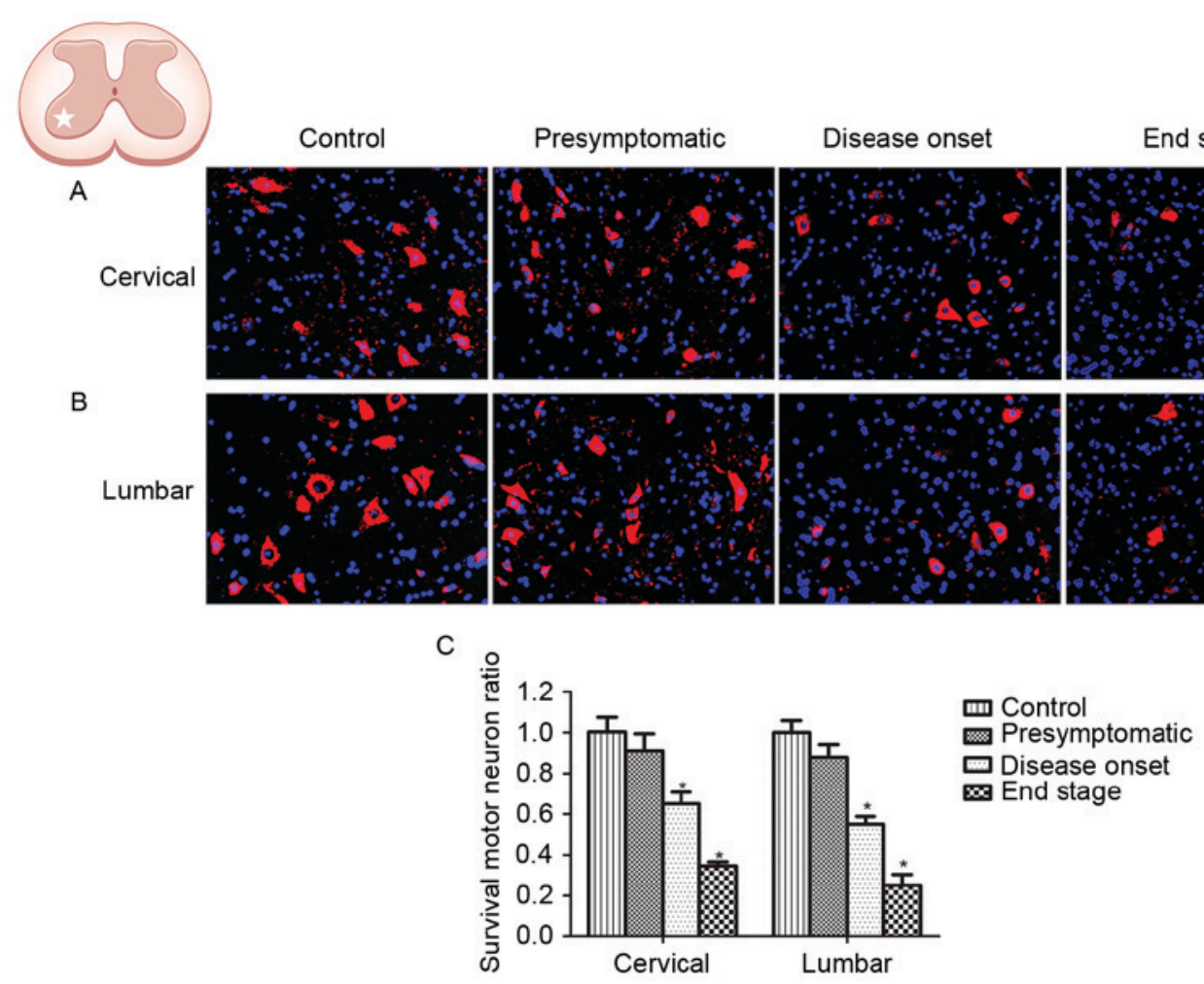

Figure 1. Degeneration of motor neurons in the cervical and lumbar ventral horn of SOD1G93A mice. (A) Representative images exhibiting the survival of ChAT-positive neurons in the cervical ventral horn. (B) Representative images exhibiting the survival of ChAT-positive neurons in the lumbar ventral horn. (C) ChAT-positive motor neurons were significantly decreased at the disease onset and end stages. ${ }^{*} \mathrm{P}<0.05$ vs. wild type control (one-way analysis of variance; $\mathrm{n}=5$ animals/group). Scale bar, $100 \mu \mathrm{m}$. SOD1, superoxide dismutase 1; ChAT, choline O-acetyltransferase.

Microsystems, Inc., Buffalo Grove, IL, USA). In order to measure regional GFAP expression, regions were uniformly thresholded and the area coverage of GFAP immunoreactivity (as a percentage of the whole region area) was measured. In order to evaluate global AQP4 expression levels, the mean AQP4 immunofluorescence intensity was measured within each region. In order to measure AQP4 polarity, the area of the image with a pixel intensity greater than or equal to that of the perivascular endfeet was measured (value expressed as a percentage of total field of view), according to a previous study (22). AQP4 polarization was characterized within the astrocytic endfeet associated with the soma in the spinal cord. A total of five randomly selected sections/animal in each group were analyzed in a blinded manner by two investigators using ImageJ version 1.50b bundled with Java 1.8.0_60 software (National Institutes of Health).

Evaluation of GLT-1 expression in the lumbar spinal cord. In order to measure the alteration in GLT-1 expression in the ventral horn of the lumbar spinal cord between WT and SOD1G93A mice at the end stage, imaging was conducted at x20 objective power using the fluorescence microscope. The area of GLT-1 immunoreactivity was expressed as a percentage of the overall field of view. A total of five randomly selected sections/animal in each group were analyzed in a blinded manner by two investigators using ImageJ software.

Statistical analysis. Data are presented as the mean \pm standard error of the mean. Comparisons between different groups were performed using one-way analysis of variance followed by
Bonferroni's post hoc test, and using a Student's t-test between two groups. All data were processed using SPSS software (version 20.0; IBM Corp., Armonk, NY, USA). P<0.05 was considered to indicate a statistically significant difference.

\section{Results}

Motor neuron degeneration in SOD1G93A mice. ChAT is considered to be a marker of motor neurons. In order to verify the onset and progression of spinal motor neuron loss in ALS, the survival of ChAT-positive neurons located in the ventral horn of SOD1G93A mice was investigated at the presymptomatic, disease onset and end stages. In order to assess disease progression, motor neuron loss was measured in the cervical and lumbar spinal cord at the same stage. No difference in the number of ChAT-positive cells was observed in the spinal and lumbar ventral horn between WT control mice and SOD1G93A mice at the presymptomatic stage (Fig. 1). The number of ChAT-positive neurons in the cervical and lumbar spinal cord of SOD1G93A mice was decreased at disease onset, when motor deficits presented; $67.7 \%$ ChAT-positive neurons survived in the cervical ventral horn, and 54.7\% ChAT-positive neurons survived in the lumbar ventral horn, compared with the control (Fig. 1). The number of ChAT-positive neurons was observed to be further decreased at the end stage, with 34.9 and $25.0 \%$ ChAT-positive neurons in the spinal and lumbar ventral horn, respectively (Fig. 1). Although SOD1G93A mice exhibit motor deficits primarily in the hind limbs, the results of the present study demonstrated that motor neuron loss occurred synchronously in the cervical and lumbar spinal cord, 


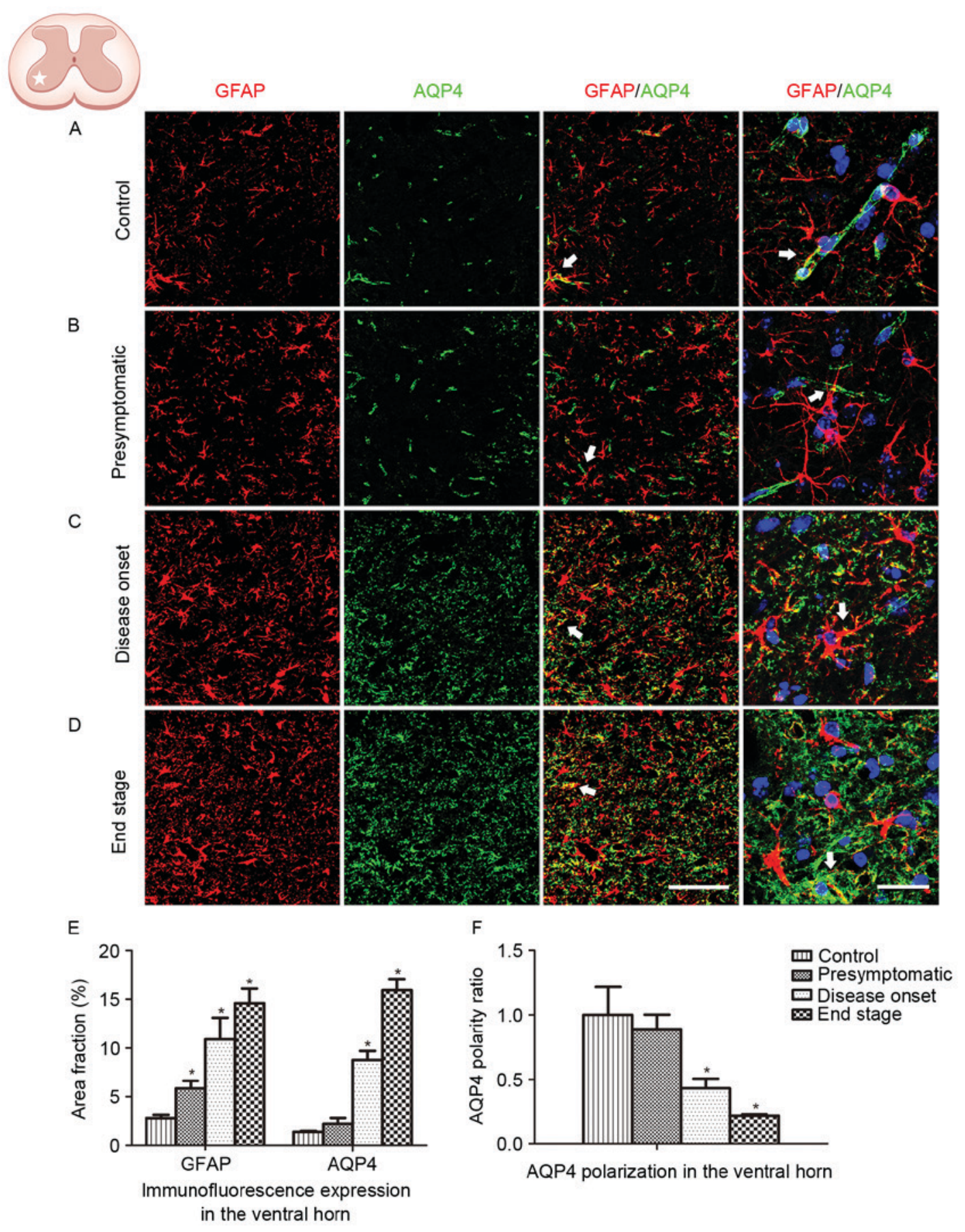

Figure 2. GFAP and AQP4 expression and localization in the ventral horn of the lumbar spinal cord. (A) GFAP and AQP4 expression and localization in wild type control animals. (B) GFAP and AQP4 expression and localization in SOD1G93A mice at the presymptomatic stage. (C) GFAP and AQP4 expression and localization in SOD1G93A mice at the disease onset stage. (D) GFAP and AQP4 expression and localization in SOD1G93A mice at the end stage. The arrows indicate areas of positive AQP4 staining. Scale bars, $150 \mu \mathrm{m}$ for the left three columns and $75 \mu \mathrm{m}$ for the far right column. (E) GFAP and AQP4 expression was quantified at the presymptomatic, disease onset and end stages using the area fraction (percentage of GFAP or AQP4 immunoreactivity in the overall field). Increased global expression of GFAP and AQP4 was observed as the disease progressed. (F) AQP4 polarization decreased as the disease progressed. ${ }^{*} \mathrm{P}<0.05$ vs. wild type control (one-way analysis of variance; $\mathrm{n}=5$ animals/group). GFAP, glial fibrillary acidic protein; AQP4, aquaporin-4; SOD1, superoxide dismutase 1 .

indicating that the pathological condition was widespread in the ALS model animals.

AQP4 overexpression and depolarization in SOD1G93A mice. GFAP and AQP4 expression in the lumbar ventral horn was analyzed in the present study (Fig. 2). An increased number of GFAP-positive cells, coupled with swelling cell morphology, indicates astrocyte activation. In the present study, no astrocyte activation was observed in WT control animals (Fig. 2A). Astrocyte activation was detected at the presymptomatic stage, and increased in the subsequent disease onset and end stages in the ALS model animals (Fig. 2B-D). Persistent reactive astrocytes with swollen cell bodies were observed at the disease onset and end stages. Global AQP4 expression was increased as 


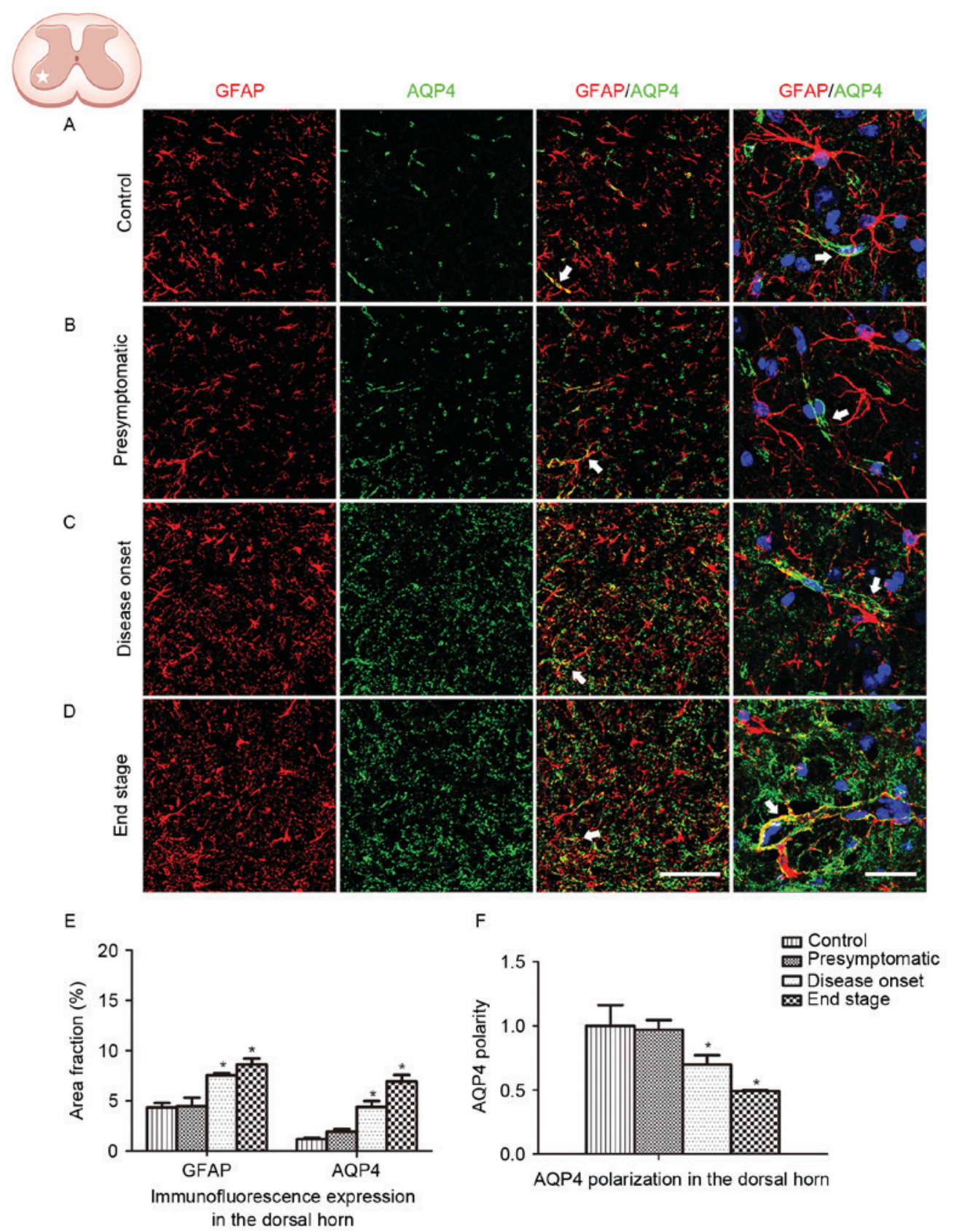

Figure 3. GFAP and AQP4 expression and localization in the ventral horn of the cervical spinal cord. (A) GFAP and AQP4 expression and localization in control animals. (B) GFAP and AQP4 expression and localization in SOD1G93A mice at the presymptomatic stage. (C) GFAP and AQP4 expression and localization in SOD1G93A mice at the disease onset stage. (D) GFAP and AQP4 expression and localization in SOD1G93A mice at the end stage. The arrows indicate areas of positive AQP4 staining. Scale bars, $150 \mu \mathrm{m}$ for the left three columns and $75 \mu \mathrm{m}$ for the far right column. (E) GFAP and AQP4 expression was quantified at the presymptomatic, disease onset and end stages using the area fraction (percentage of GFAP or AQP4 immunoreactivity in the overall field). Increased global expression of GFAP and AQP4 was observed as the disease progressed. (F) AQP4 polarization decreased as the disease progressed. ${ }^{*} \mathrm{P}<0.05$ vs. wild type control (one-way analysis of variance; $\mathrm{n}=5$ animals/group). GFAP, glial fibrillary acidic protein; AQP4, aquaporin-4; SOD1, superoxide dismutase 1 .

the disease progressed. Co-localization of GFAP labeling with AQP4 labeling was observed (Fig. 2E). AQP4 expression in WT animals and SOD1G93A mice at the presymptomatic stage was predominantly located in the astrocytic endfeet, which exhibited a cord-like shape (Fig. 2A and B). However, as the disease progressed in the SOD1G93A mice, AQP4 expression was not restricted to astrocytic endfeet domains. At high magnification, AQP4 expression was observed to shift to the membranes of swollen astrocytic soma at the disease onset stage and the end stage, indicating a loss of AQP4 polarization (Fig. 2C, D and F).
The spinal ventral horn, where motor neurons are located, is the most affected area in ALS. In order to further depict the alteration in GFAP and AQP4 expression in ALS, protein expression in the dorsal horn was additionally analyzed. Similar GFAP and AQP4 expression profiles were observed in the dorsal horn of the lumbar spinal cord, with AQP4 expression increasing and shifting to the membranes of astrocytic soma as the disease progressed (Fig. 3). AQP4 overexpression and depolarization were additionally observed in the cervical spinal cord of SOD1G93A mice (data not presented), indicating 


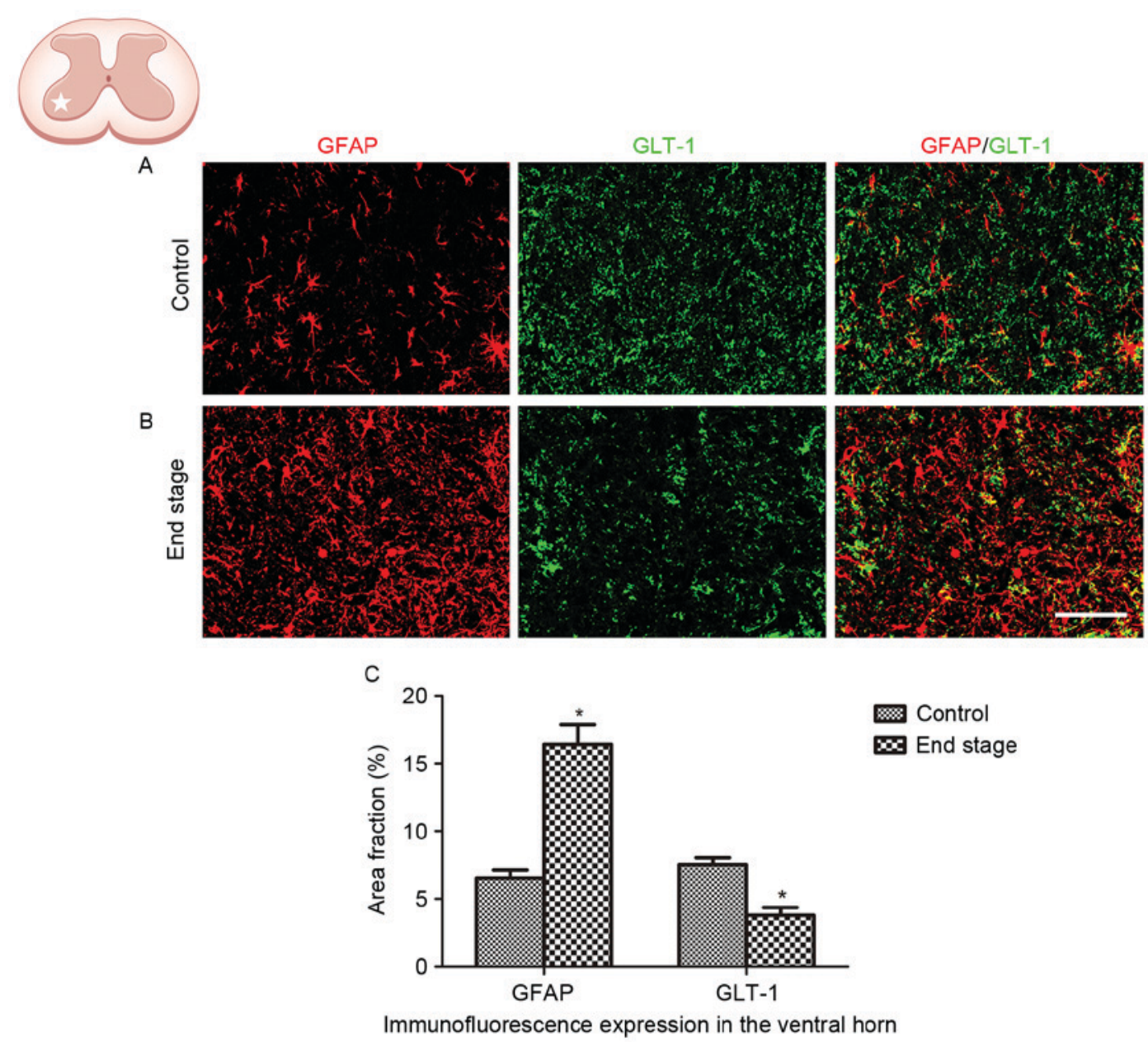

Figure 4. GLT-1 expression in astrocytes, in the lumbar ventral horn of SOD1G93A mice. (A) Representative images exhibiting GFAP and GLT-1 staining in wild type control mice. (B) Representative images exhibiting GFAP and GLT-1 staining in SOD1G93A mice at the end stage. Scale bar, $75 \mu \mathrm{m}$. (C) Quantification demonstrated that GLT-1 expression decreased in the ventral horn at the end stage of disease in SOD1G93A mice. ${ }^{*} \mathrm{P}<0.05$ vs. wild type control (Student's t test; $\mathrm{n}=5$ animals/group). GLT-1, glutamate transporter 1; GFAP, glial fibrillary acidic protein; SOD1, superoxide dismutase 1.

that the loss of AQP4 polarization existed throughout the spinal cord in the ALS animals.

Decreased GLT-1 expression in SODIG93A mice. The alteration in GLT-1 expression in ALS was analyzed in the present study. A significant decrease in GLT-1 immunoreactivity was observed in the lumbar ventral horn of SOD1G93A mice at the end stage, compared with the WT control (Fig. 4; P<0.01).

\section{Discussion}

The homeostasis of the CNS and the stability of its function are dependent on the integrity of the BBB and the balance of water, glutamate and ions. A major contributing factor to the maintenance of these homeostatic conditions in the vicinity of active neurons is the expression of AQP4, GLT-1 and Kir4.1, which are co-expressed in the membrane of astrocytes. The association between BBB and BSCB disruption, and astrocyte dysfunction in ALS, has been demonstrated by numerous studies (4-10). In the present study, using SOD1G93A mutant mice, the association between motor neuron survival, astrocyte activation, AQP4 dysregulation and the alteration in GLT1 expression in ALS was investigated.
Astrocyte activation accompanied by motor neuron degeneration was observed in the spinal cord of SOD1G93A mice. Astrocyte activation was observed at the presymptomatic stage when motor deficits were not detected, suggesting that the glial pathophysiological response in ALS is initiated prior to the onset of clinical symptoms. The present study demonstrated the overexpression of AQP4, and additionally demonstrated the loss of AQP4 polarization in SOD1G93A mice. In physiological conditions, AQP4 exhibits a polarized distribution, with increased density in perivascular astrocytic endfeet membranes compared with other membrane domains. AQP4 polarization may be an important facilitator of the function of AQP4 in maintaining water homeostasis; a hypothesis that was supported by a previous study using $\alpha$-syntrophin knockout mice. The protein $\alpha$-syntrophin is required in order for AQP4 to anchor at the perivascular endfeet membrane. In $\alpha$-syntrophin knockout mice, AQP4 localization was shifted away from the endfeet domains, while overall AQP4 expression was not altered, which resulted in impaired AQP4-dependent $\mathrm{K}^{+}$clearance (24) and cerebral edema following cerebral ischemia (25). A previous study using AQP4 knockout mice demonstrated that perivascular AQP4 served a role in regulating extracellular volume dynamics by controlling water flux in the mammalian brain (26). 
Therefore, the loss of perivascular AQP4 may initiate a number of pathophysiological processes. Redistribution of AQP4 was observed to counteract early edema formation in an animal model of brain infarction (27). In an animal model of mesial temporal lobe epilepsy, the depolarization of perivascular AQP4 was demonstrated to precede the development of chronic seizures (15). A loss of AQP4 polarization has additionally been observed in a mouse model of Alzheimer's disease (28).

Due to the physiological role of AQP4, it has been demonstrated to serve as an important component of the paravascular pathway that assist in the clearance of $\beta$-amyloid, and other molecules, from the brain parenchyma (29). Additionally, AQP4 depolarization has been observed in the aging brain, with impaired paravascular clearance (30). In an animal model of traumatic brain injury, an elevated global level of AQP4 protein with a redistribution manner of depolarization was observed in the acute stage of injury, leading to the formation and resolution of cerebral edema (31). However, the sustained loss of AQP4 perivascular polarization impaired the paravascular pathway clearance of interstitial solutes from the brain, contributing to microtubule-associated protein tau aggregation and neurodegeneration, in the chronic phase following traumatic brain injury (16). It is therefore likely that polarized AQP4 serves a role in clearance of interstitial solutes from the brain.

In the present study, perivascular localization of AQP4 in astrocytic endfeet was observed in the spinal cord prior to disease onset, which is consistent with previous studies $(19,20,32)$. Depolarized AQP4 expression was observed to occur in line with the progression of ALS. AQP4 depolarization may be a pathological factor associated with the onset and progression of ALS. Sustained depolarization of AQP4 impairs the function of maintaining water balance in the spinal cord, leading to swelling and malformation of astrocytes and interfering with neuronal function in ALS; however, the aggregation of misfolded mutant SOD1 proteins is known to be a cause of ALS. It is hypothesized that the loss of AQP4 polarization has a negative impact on the clearance of interstitial solutes, including mutant SOD1 proteins, from the spinal cord, and thereby contributes to the aggregation of misfolded mutant SOD1 proteins in ALS. Therefore, further investigation is required to investigate the association between the loss of AQP4 polarization and the aggregation of misfolded mutant SOD1 proteins.

An additional molecule expressed in astrocytes is GLT-1, a glutamate transporter that is responsible for the majority of functional glutamate uptake in the CNS (33). It has been demonstrated that one of the major causes of motor neuron degeneration in ALS is excitotoxicity due to dysregulation of extracellular glutamate homeostasis (34). Compromised glutamate uptake, due to decreased expression and aberrant functioning of GLT-1, has been observed in astrocytes of the spinal cord and motor cortex in patients with ALS $(35,36)$ and SOD1G93A rodents (37). In the present study, decreased expression of GLT-1 in the spinal cord in SOD1G93A mice was observed, which is consistent with the previous studies (38). Downregulated GLT-1 expression in astrocytes and impaired uptake of glutamate were observed in AQP4-deficient mice (38), indicating that AQP4 serves a role in regulating the expression and function of GLT-1. Glutamate has been demonstrated to increase AQP4 water permeability in astrocytes (39). A number of studies into various neurological disorders have demonstrated that AQP4 and GLT-1 exist in astrocytic membranes as a functional complex (40-43). It is hypothesized that the overexpression and depolarization of AQP4 leads to abnormal functioning, which may therefore lead to the decreased expression and aberrant functioning of GLT-1. The association between AQP4 and GLT-1 may result in impairment of the balance between glutamate and water transport, dysregulation of neuronal activity, and excitotoxic neuronal dysfunction. Further studies are required to elucidate the interaction between AQP4 and GLT-1.

In the present study, SOD1G93A mice were used as a model to investigate the role of AQP4 in the pathological development of ALS. Global overexpression, with decreased polarized astrocytic localization of AQP4, was observed in the spinal cord, indicating an impaired function of AQP4 in ALS. The results of the present study demonstrated that AQP4 depolarization is a widespread pathological condition and may contribute to motor neuron degeneration in ALS.

\section{Acknowledgements}

The present study was supported by grants from the Guangzhou Science and Technology Project (grant no. 2014J4500031), the Guangdong Science and Technology Project (grant nos. 2013B021800274, 2014B030301035 and 2015B050501003), the Major Cultivation and Interdisciplinary Project of Sun Yat-Sen University (grant no. 15ykjc15b) and the Macau Science and Technology Development Fund (grant no. 063/2015/A2).

\section{References}

1. Kiernan MC, Vucic S, Cheah BC, Turner MR, Eisen A, Hardiman O, Burrell JR and Zoing MC: Amyotrophic lateral sclerosis. Lancet 377: 942-955, 2011

2. Rosen DR, Siddique T, Patterson D, Figlewicz DA, Sapp P, Hentati A, Donaldson D, Goto J, O'Regan JP, Deng HX, et al: Mutations in $\mathrm{Cu} / \mathrm{Zn}$ superoxide dismutase gene are associated with familial amyotrophic lateral sclerosis. Nature 362: 59-62, 1993.

3. Mancuso R and Navarro X: Amyotrophic lateral sclerosis: Current perspectives from basic research to the clinic. Prog Neurobiol 133: 1-26, 2015.

4. Garbuzova-Davis S, Hernandez-Ontiveros DG, Rodrigues MC, Haller E, Frisina-Deyo A, Mirtyl S, Sallot S, Saporta S, Borlongan CV and Sanberg PR: Impaired blood-brain/spinal cord barrier in ALS patients. Brain Res 1469: 114-128, 2012.

5. Winkler EA, Sengillo JD, Sullivan JS, Henkel JS, Appel SH and Zlokovic BV: Blood-spinal cord barrier breakdown and pericyte reductions in amyotrophic lateral sclerosis. Acta Neuropathol 125: 111-120, 2013.

6. Yamadera M, Fujimura H, Inoue $\mathrm{K}$, Toyooka K, Mori C, Hirano $\mathrm{H}$ and Sakoda S: Microvascular disturbance with decreased pericyte coverage is prominent in the ventral horn of patients with amyotrophic lateral sclerosis. Amyotroph Lateral Scler Frontotemporal Degener 16: 393-401, 2015.

7. Sasaki S: Alterations of the blood-spinal cord barrier in sporadic amyotrophic lateral sclerosis. Neuropathology 35: 518-528, 2015.

8. Garbuzova-Davis S, Haller E, Saporta S, Kolomey I, Nicosia SV and Sanberg PR: Ultrastructure of blood-brain barrier and blood-spinal cord barrier in SOD1 mice modeling ALS. Brain Res 1157: 126-137, 2007.

9. Garbuzova-Davis S, Saporta S, Haller E, Kolomey I, Bennett SP, Potter $\mathrm{H}$ and Sanberg PR: Evidence of compromised blood-spinal cord barrier in early and late symptomatic SOD1 mice modeling ALS. PLoS One 2: e1205, 2007.

10. Winkler EA, Sengillo JD, Sagare AP, Zhao Z, Ma Q, Zuniga E, Wang Y, Zhong Z, Sullivan JS, Griffin JH, et al: Blood-spinal cord barrier disruption contributes to early motor-neuron degeneration in ALS-model mice. Proc Natl Acad Sci USA 111: E1035-E1042, 2014. 
11. Blackburn D, Sargsyan S, Monk PN and Shaw PJ: Astrocyte function and role in motor neuron disease: A future therapeutic target? Glia 57: 1251-1264, 2009.

12. Nicchia GP, Nico B,Camassa LM,Mola MG, Loh N, DermietzelR, Spray DC, Svelto M and Frigeri A: The role of aquaporin-4 in the blood-brain barrier development and integrity: Studies in animal and cell culture models. Neuroscience 129: 935-945, 2004.

13. Tomás-Camardiel M, Venero JL, Herrera AJ, De Pablos RM, Pintor-Toro JA, Machado A and Cano J: Blood-brain barrier disruption highly induces aquaporin- 4 mRNA and protein in perivascular and parenchymal astrocytes: Protective effect by estradiol treatment in ovariectomized animals. J Neurosci Res 80: 235-246, 2005.

14. Li S, Hu X, Zhang M, Zhou F, Lin N, Xia Q, Zhou Y, Qi W, Zong Y, Yang $\mathrm{H}$ and Wang T: Remote ischemic post-conditioning improves neurological function by $\mathrm{AQP} 4$ down-regulation in astrocytes. Behav Brain Res 289: 1-8, 2015.

15. Alvestad S, Hammer J, Hoddevik EH, Skare $\varnothing$, Sonnewald U, Amiry-Moghaddam M and Ottersen OP: Mislocalization of AQP4 precedes chronic seizures in the kainate model of temporal lobe epilepsy. Epilepsy Res 105: 30-41, 2013.

16. Iliff JJ, Chen MJ, Plog BA, Zeppenfeld DM, Soltero M, Yang L, Singh I, Deane R and Nedergaard M: Impairment of glymphatic pathway function promotes tau pathology after traumatic brain injury. J Neurosci 34: 16180-16193, 2014.

17. Xu Z, Xiao N, Chen Y, Huang H, Marshall C, Gao J, Cai Z, Wu T, $\mathrm{Hu}$ G and Xiao M: Deletion of aquaporin-4 in APP/PS1 mice exacerbates brain $A \beta$ accumulation and memory deficits. Mol Neurodegener 10: 58, 2015.

18. Nesic O, Lee J, Ye Z, Unabia GC, Rafati D, Hulsebosch CE and Perez-Polo JR: Acute and chronic changes in aquaporin 4 expression after spinal cord injury. Neuroscience 143: 779-792, 2006.

19. Nicaise C, Soyfoo MS, Authelet M, De Decker R, Bataveljic D, Delporte C and Pochet R: Aquaporin-4 overexpression in rat ALS model. Anat Rec (Hoboken) 292: 207-213, 2009.

20. Bataveljic D, Nikolić L, Milosević M, Todorović N and Andjus PR: Changes in the astrocytic aquaporin-4 and inwardly rectifying potassium channel expression in the brain of the amyotrophic lateral sclerosis SOD1(G93A) rat model. Glia 60: 1991-2003, 2012.

21. Cui Y, Masaki K, Yamasaki R, Imamura S, Suzuki SO, Hayashi S, Sato S, Nagara Y, Kawamura MF and Kira J: Extensive dysregulations of oligodendrocytic and astrocytic connexins are associated with disease progression in an amyotrophic lateral sclerosis mouse model. J Neuroinflammation 11: 42, 2014.

22. Gurney ME, Pu H, Chiu AY, Dal Canto MC, Polchow CY, Alexander DD, Caliendo J, Hentati A, Kwon YW, Deng HX, et al: Motor neuron degeneration in mice that express a human $\mathrm{Cu}, \mathrm{Zn}$ superoxide dismutase mutation. Science 264: 1772-1775, 1994.

23. Weydt P, Hong SY, Kliot M and Möller T: Assessing disease onset and progression in the SOD1 mouse model of ALS. Neuroreport 14: 1051-1054, 2003.

24. Amiry-Moghaddam M, Williamson A, Palomba M, Eid T, de Lanerolle NC, Nagelhus EA, Adams ME, Froehner SC, Agre P and Ottersen OP: Delayed K+ clearance associated with aquaporin-4 mislocalization: Phenotypic defects in brains of alpha-syntrophin-null mice. Proc Natl Acad Sci USA 100: 13615-13620, 2003.

25. Amiry-Moghaddam M, Otsuka T, Hurn PD, Traystman RJ, Haug FM, Froehner SC, Adams ME, Neely JD, Agre P, Ottersen OP and Bhardwaj A: An alpha-syntrophin-dependent pool of AQP4 in astroglial end-feet confers bidirectional water flow between blood and brain. Proc Natl Acad Sci USA 100: 2106-2111, 2003.

26. Haj-Yasein NN, Jensen V, Østby I, Omholt SW, Voipio J, Kaila K, Ottersen OP, Hvalby $\varnothing$ and Nagelhus EA: Aquaporin-4 regulates extracellular space volume dynamics during high-frequency synaptic stimulation: A gene deletion study in mouse hippocampus. Glia 60: 867-874, 2012.
27. Steiner E, Enzmann GU, Lin S, Ghavampour S, Hannocks MJ, Zuber B, Rüegg MA, Sorokin L and Engelhardt B: Loss of astrocyte polarization upon transient focal brain ischemia as a possible mechanism to counteract early edema formation. Glia 60: 1646-1659, 2012.

28. Yang J,Lunde LK, Nuntagij P, Oguchi T, Camassa LM, Nilsson LN, Lannfelt L, Xu Y, Amiry-Moghaddam M, Ottersen OP and Torp R: Loss of astrocyte polarization in the tg-ArcSwe mouse model of Alzheimer's disease. J Alzheimers Dis 27: 711-722, 2011.

29. Iliff JJ, Wang M, Liao Y, Plogg BA, Peng W, Gundersen GA, Benveniste H, Vates GE, Deane R, Goldman SA, et al: A paravascular pathway facilitates CSF flow through the brain parenchyma and the clearance of interstitial solutes, including amyloid $\beta$. Sci Transl Med 4: 147ra111, 2012.

30. Kress BT, Iliff JJ, Xia M, Wang M, Wei HS, Zeppenfeld D, Xie L, Kang H, Xu Q, Liew JA, et al: Impairment of paravascular clearance pathways in the aging brain. Ann Neurol 76: 845-861, 2014.

31. Ren Z, Iliff JJ, Yang L, Yang J, Chen X, Chen MJ, Giese RN, Wang B, Shi X and Nedergaard M: 'Hit \& Run' model of closed-skull traumatic brain injury (TBI) reveals complex patterns of post-traumatic AQP4 dysregulation. J Cereb Blood Flow Metab 33: 834-845, 2013.

32. Oklinski MK, Lim JS, Choi HJ, Oklinska P, Skowronski MT and Kwon TH: Immunolocalization of water channel proteins AQP1 and AQP4 in rat spinal cord. J Histochem Cytochem 62: 598-611, 2014.

33. Maragakis NJ and Rothstein JD: Glutamate transporters: Animal models to neurologic disease. Neurobiol Dis 15: 461-473, 2004.

34. Maragakis NJ and Rothstein JD: Mechanisms of disease: Astrocytes in neurodegenerative disease. Nat Clin Pract Neurol 2: 679-689, 2006.

35. Rothstein JD, Martin LJ and Kuncl RW: Decreased glutamate transport by the brain and spinal cord in amyotrophic lateral sclerosis. N Engl J Med 326: 1464-1468, 1992.

36. Rothstein JD, Van Kammen M, Levey AI, Martin LJ and Kuncl RW: Selective loss of glial glutamate transporter GLT-1 in amyotrophic lateral sclerosis. Ann Neurol 38: 73-84, 1995.

37. Howland DS, Liu J, She Y, Goad B, Maragakis NJ, Kim B, Erickson J, Kulik J, DeVito L, Psaltis G, et al: Focal loss of the glutamate transporter EAAT2 in a transgenic rat model of SOD1 mutant-mediated amyotrophic lateral sclerosis (ALS). Proc Natl Acad Sci USA 99: 1604-1609, 2002.

38. Zeng XN, Sun XL, Gao L, Fan Y, Ding JH and Hu G: Aquaporin-4 deficiency down-regulates glutamate uptake and GLT-1 expression in astrocytes. Mol Cell Neurosci 34: 34-39, 2007.

39. Gunnarson E, Zelenina M, Axehult G, Song Y, Bondar A, Krieger P, Brismar H, Zelenin S and Aperia A: Identification of a molecular target for glutamate regulation of astrocyte water permeability. Glia 56: 587-596, 2008.

40. Hinson SR, Roemer SF, Lucchinetti CF, Fryer JP, Kryzer TJ, Chamberlain JL, Howe CL, Pittock SJ and Lennon VA: Aquaporin-4-binding autoantibodies in patients with neuromyelitis optica impair glutamate transport by down-regulating EAAT2. J Exp Med 205: 2473-2481, 2008.

41. Yang J, Li MX, Luo Y, Chen T, Liu J, Fang P, Jiang B, Hu ZL, Jin Y, Chen JG and Wang F: Chronic ceftriaxone treatment rescues hippocampal memory deficit in AQP4 knockout mice via activation of GLT-1. Neuropharmacology 75: 213-222, 2013.

42. Mogoanta L, Ciurea M, Pirici I, Margaritescu C, Simionescu C, Ion DA and Pirici D: Different dynamics of aquaporin 4 and glutamate transporter-1 distribution in the perineuronal and perivascular compartments during ischemic stroke. Brain Pathol 24: 475-493, 2014.

43. Geis C, Ritter C, Ruschil C, Weishaupt A, Grünewald B, Stoll G, Holmoy T, Misu T, Fujihara K, Hemmer B, et al: The intrinsic pathogenic role of autoantibodies to aquaporin 4 mediating spinal cord disease in a rat passive-transfer model. Exp Neurol 265: 8-21, 2015. 